\title{
Isolated tibial nerve injury: a rare presentation
}

\author{
Rahul Krishnarao Patil'1, Prashant Verkey'1, Harshal Patil', Deepesh Manoharan'1 \\ ${ }^{1}$ Jubilee Institute for the Surgery of Hand Aesthetic and Microvascular Surgery, Jubilee Mission Hospital, Thrissur 680005, Kerala, India. \\ ${ }^{2}$ Department of General Surgery, Jubilee Mission Hospital, Thrissur 680005, Kerala, India.
}

Address for correspondence: Dr. Rahul Krishnarao Patil, Sparsh Hospital, Infantry Road, Opp. Commissioners Office, Bengaluru 560001, Karnataka, India. E-mail: doctrahul@yahoo.co.in

\begin{abstract}
Tibial nerve injury is rare and is always associated with other injuries due to its close association with the other structures. We present a rare case of isolated injury to the tibial nerve where the nerve was avulsed from the middle third of the leg, but all other structures were intact. The nerve was reconstructed with sural nerve grafts. The patient recovered sensation of the sole twelve months following the reconstruction and was able to maintain a normal gait and is living normal life. The results of nerve repairs in lower limbs in general have been poor. The treatment options for such an interesting case are discussed along with the management and outcome of the presented patient.
\end{abstract}

Key words:

Nerve injuries in the lower limb, nerve reconstruction in lower extremity, tibial nerve injury

\section{INTRODUCTION}

The tibial nerve lies between the superficial and the deep muscles of the posterior compartment of the leg. It is well-protected from direct trauma due to this thick cover of muscles. In the lowermost part of the leg and ankle, the nerve is relatively superficial but is guarded anteriorly by the posterior surface of the medial malleolus, superficially by the flexor retinaculum and posteriorly by the Achilles's tendon. This protected location makes isolated injury to the tibial nerve is very uncommon. Even in cases of open fractures and associated vascular injuries of the lower extremity complete transaction of the tibial nerve is rare. ${ }^{[1]}$ Injuries to the sciatic and common peroneal nerves are more common due to their vulnerable position. ${ }^{[2,3]}$ Most of the available literature on peripheral nerve injuries in the lower extremity has documented the results and the treatment options for peroneal and sciatic nerve injuries.

Other common causes of lower limb neuropathy are diabetic neuropathy ${ }^{[4]}$ and compression neuropathies. ${ }^{[5]}$ Tibial nerve involvement is more common in these chronic

\begin{tabular}{|l|l|}
\hline \multicolumn{2}{|c|}{ Access this article online } \\
\hline Quick Response Code: & Website: \\
\hline & www.parjournal.net \\
\cline { 2 - 2 } & \\
\hline
\end{tabular}

conditions. The common end result of the tibial nerve injury or neuropathy around the ankle is loss of sensations of the plantar foot, vasomotor changes due to lack of auto-regulation, subsequently leading to callosities and recurrent ulcerations and paralysis of the intrinsic muscles of foot leading to toe deformities.

We report a very unusual presentation of isolated posterior tibial nerve injury following a road traffic accident. Our search failed to reveal any similar case reported in the English literature.

\section{CASE REPORT}

A written consent was obtained from the patient and her attendants. A 15-year-old girl sustained injury to her left leg following road traffic accident. Though the exact mechanism of injury could not be elicited, she remembered falling down from her two-wheeler after it collided with a car. She sustained a small puncture wound over the lower posterior leg and was referred to our hospital after the first aid at a local hospital. On examination, she had a penetrating wound over the Achilles tendon with some soft tissue mass avulsed through the tear in the Achilles tendon [Figure 1]. The avulsed soft tissue was tender on touch. She had a lack of sensation over the plantar foot, and the foot was warm. The skin texture and turgor were found to be normal. With a provisional diagnosis of tibial nerve injury, the wound was explored. The soft tissue avulsed and protruding through the tendon was the tibial nerve [Figure 2a]. While the posterior tibial vessels and the flexor tendons were intact. 
The leg was explored to find the proximal extent of injury. The nerve was found to be avulsed from the middle third as could be predicted from the length of avulsed segment [Figure $2 \mathrm{~b}$ ]. In view of the avulsion component and the force involved (site of rupture was around $8 \mathrm{~cm}$ from the site of a puncture wound), we decided to wait for 3 weeks before going in for definitive reconstruction. The nerve ends were tacked for future identification, and the wound was closed.

After 3 weeks, the wound was re-explored. The tibial nerve was traced proximally into unscarred tissues. After sectioning the nerve at this level, it was observed under microscope for healthy fascicles. The same procedure was followed in the distal leg to secure a healthy distal end of the nerve. The final defect of around $9.5 \mathrm{~cm}$ was reconstructed with 3 sural nerve cable grafts of $10 \mathrm{~cm}$ each [Figure 3] harvested from the contra-lateral lower extremity (the diameter of the tibial nerve at the proximal and distal end was suitable to accommodate 3 cables of a sural nerve graft). The patient was instructed regarding the care of the insensate foot, to avoid walking bare foot and perform regular inspection of the plantar foot.

She has completed 3 years of follow-up and the recovery so far is satisfactory. Except mild hallowing of the instep

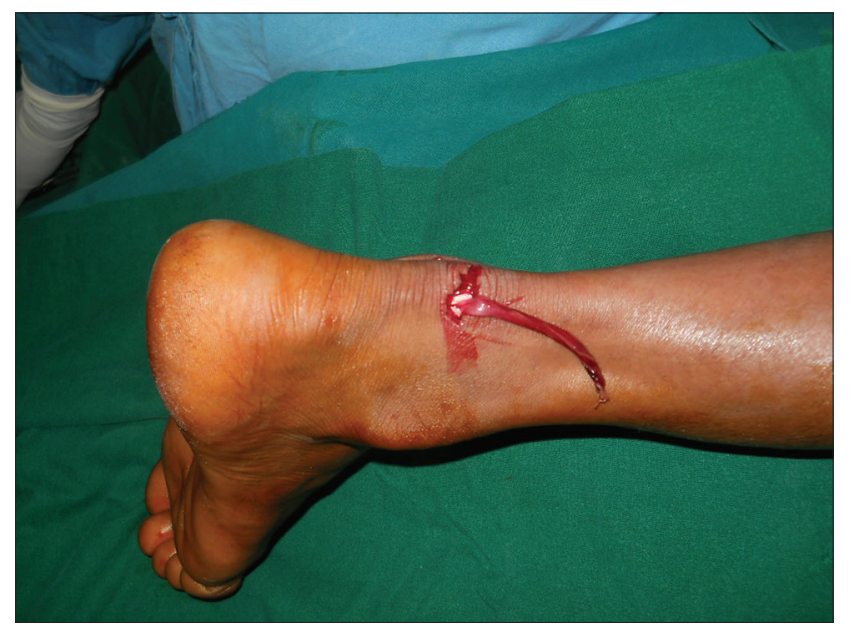

Figure 1: Patient presenting with a small wound over the posterior aspect of the heel with soft tissue protruding through tendoachillis

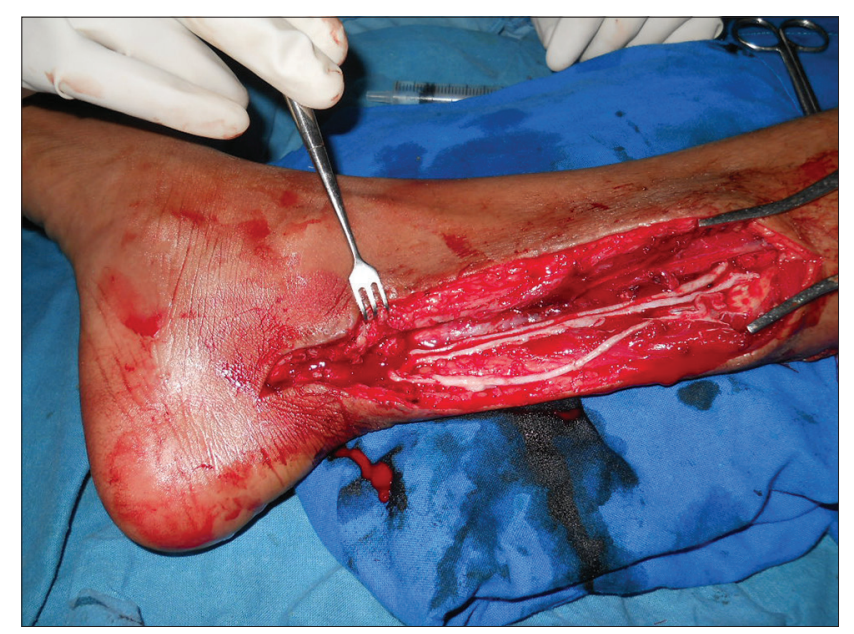

Figure 3: Photograph taken during secondary reconstruction. The nerve being reconstructed with sural nerve cable grafts from the same leg area, there have been no deformities [Figure 4]. Patient has regained sensation over the heel and the instep area, and the progressive Hoffmann-Tinnel signs had reached the forefoot area one and a half year after the surgery. Figure 5 shows an easy way to monitor the progressive Tinnel signs in the patient's record. The patient has been using her limb during all her daily activities and was actively participating in sports. She has been following the instructions and did not have any pressure related complication over the plantar foot. For the past 3 years of follow-up, she has normal movements of the foot and the toes, no deformities and has recovered good protective plantar sensations.

\section{DISCUSSION}

Well recognized and documented examples of nerve injuries in the lower limb are the sciatic nerve injury at the hip during posterior fracture dislocations, iatrogenic injuries during injections and peroneal nerve injury following fracture of the neck of the fibula. ${ }^{[2,3,6]}$ Injury to the posterior tibial nerve is, fortunately, very rare. ${ }^{[1,7,8]}$

Though the mechanism of injury is not exactly known in the presented case, some sharp object must have pierced the Achilles tendon to reach the nerve and due to change of the direction as the patient fell down it must have got entangled around the nerve, ultimately avulsing it.

The actual site of nerve injury was much higher than perceived site of injury, possibly a relatively fixed point like a muscular branch and is difficult to predict. ${ }^{[9]}$ This
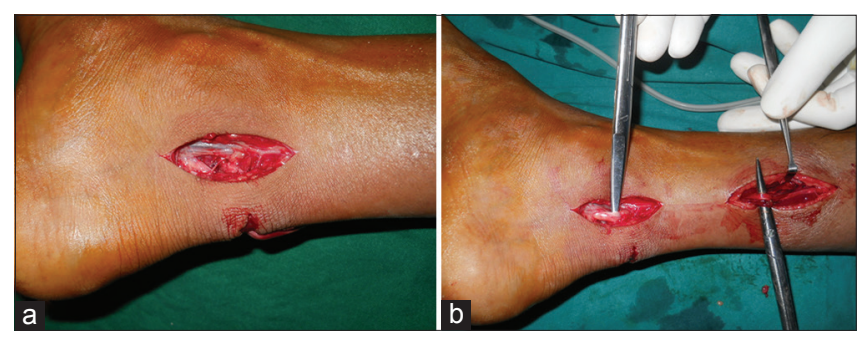

Figure 2: (a) On exploration, the soft tissue structure was found to be the posterior tibial nerve; (b) the nerve was explored in the leg and the nerve was found to have got avulsed from the middle third of the leg

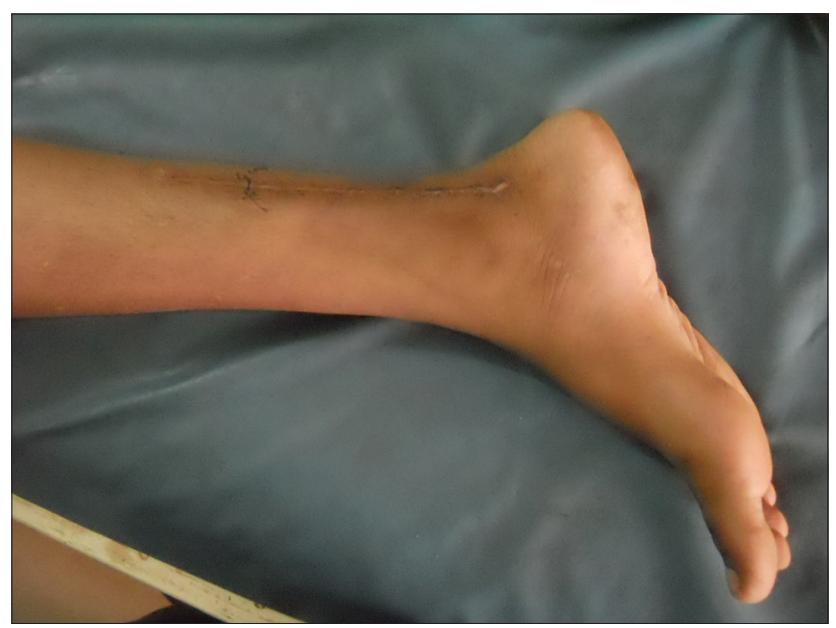

Figure 4: Picture taken after 3 weeks. The mark indicated the level of tunnels at that time. The wound has healed well 


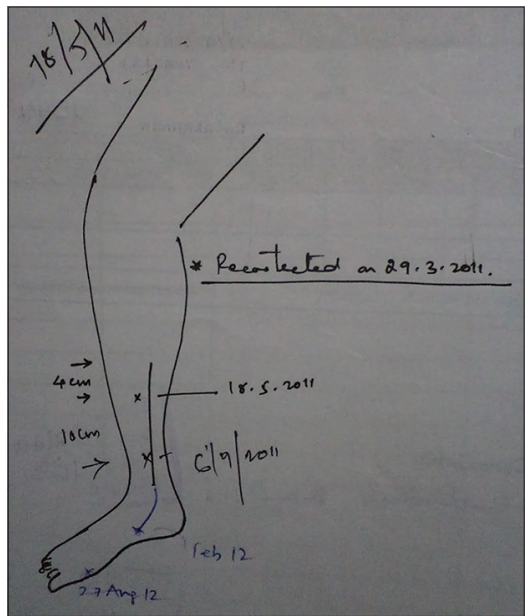

Figure 5: Picture from the progress note: showing an easy way of monitoring the recovery

can be considered analogues to brachial plexus injuries where the forces involved usually avulse the nerves from a relatively fixed point. This may also help in explaining the fact that the upper roots are either avulsed or ruptured (as they have few branches in the neck), but the lower roots are almost always avulsed in a global brachial plexus injury. Nerves are much tougher structures and coniderable force is required to avulse a nerve completely. In the presented case the nerve was completely avulsed, indicating the force involved.

Nerve repair or reconstruction should be carried out as early as possible after the injury. Children have better potential for nerve recovery and primary repair should be attempted as and when possible. For grossly contaminated wounds, injuries with extensive crushing and cases where it is difficult to know the exact extent of injury, delayed primary repair is recommended. ${ }^{[10]}$ Radical debridement up to vital axons and nerve grafting was the only chance for recovery. In our case the avulsion of the nerve and the amount of damage observed on table warranted the waiting period of 3 weeks before definitive reconstruction.

After diagnosing the injury, there were two possible ways of reconstruction of the defect/loss. One option was a primary nerve transfer ${ }^{[11,12]}$ and the other was that of nerve reconstruction with nerve grafts. ${ }^{[13]}$ The number of axons in the donors locally available (superficial peroneal and sural) are limited, and also they have only sensory fibers. On the contrary, the tibial nerve has sensory and motor fibers for the intrinsic muscles of the foot. Nerve grafting in children has better success rate than in adults. ${ }^{[14]}$ We harvested nerve grafts from contra-lateral sural nerve to keep the option of nerve transfer open if required in the future. She recovered sensations completely in around one and half years after the reconstruction, and her age was the most important factor in her recovery.

Injury to the tibial nerve in the lower leg leads to the loss of sensation at the plantar foot. Though the function of the leg muscles was preserved in this case, the insensate foot can be equally disabling due to loss of position sense and predisposition to injuries to the plantar foot. Atrophy and vasomotor changes complicate the injury. Furthermore, the paralysis of the intrinsic muscles of the foot leads to deformities over a period.

These patients need to protect their feet from injuries till they regain the protective sensations. Importance of the care of the insensate part has to be stressed during each follow-up visit. At the initial visit, the exploration of the wound for debridement and assessment of injury and the middle third of the leg for assessing the status of the proximal stump were necessary. We feel that these could have been possible through two separate incisions to decrease scarring. The nerve reconstruction also would have been possible at a later date through the same scars by tunneling the nerve grafts subcutaneously. In this case as the initial scar was present we went through the same scar for reconstruction.

In a selected and cooperative patient, nerve grafting in lower extremity can result in rewarding results.

\section{REFERENCES}

I. Waikakul S, Sakkarnkosol S, Vanadurongwan V. Vascular injuries in compound fractures of the leg with initially adequate circulation. J Bone Joint Surg $\mathrm{Br}$ 1998;80:254-8.

2. Simon JP, Van Delm I, Fabry G. Sciatic nerve palsy following hip surgery. Acta Orthop Belg 1993;59:156-62.

3. Ferraresi S, Garozzo D, Buffatti P. Common peroneal nerve injuries: results with one-stage nerve repair and tendon transfer. Neurosurg Rev 2003;26:175-9.

4. Tudhope L. Treatment of diabetic neuropathy in the lower limb. Diabet Neurop 2010;28:186-9.

5. Beltran LS, Bencardino J, Ghazikhanian V, Beltran J. Entrapment neuropathies III: Lower limb. Semin Musculoskelet Radiol 2010;14:50 I-I I.

6. Bigos SJ, Coleman SS. Foot deformities secondary to gluteal injection in infancy. J Pediatr Orthop 1984;4:560-3.

7. Brunner WG, Spencer RF. Posterior tibial nerve neurotmesis complicating a closed tibial fracture. A case report. S Afr Med J 1990;78:607-8.

8. Howard PW, Makin GS. Lower limb fractures with associated vascular injury. J Bone Joint Surg Br 1990;72:I I6-20.

9. Songcharoen P. Management of brachial plexus injury in adults. Scand J Surg 2008;97:317-23.

10. Songcharoen P. Neurotization in the treatment of brachial plexus injury. In: Omer GE, Spinner M, Van Beek AL, editors. Management of Peripheral Nerve Problems. Philadelphia: W.B. Saunders; 1998. p. 459-64.

II. Koshima I, Nanba Y, Tsutsui T, Takahashi Y. Deep peroneal nerve transfer for established plantar sensory loss. J Reconstr Microsurg 2003; 19:45। -4.

12. Gordon L, Buncke HJ. Restoration of sensation to the sole of the foot by nerve transfer. A case report. J Bone Joint Surg Am 1981;63:828-30.

13. Nunley JA, Gabel GT. Tibial nerve grafting for restoration of plantar sensation. Foot Ankle 1993; 14:489-92.

14. Senes FM, Campus R, Becchetti F, Catena N. Lower limb nerve injuries in children. Microsurgery 2007;27:32-6.

How to cite this article: Patil RK, Verkey P, Patil H, Manoharan D. Isolated tibial nerve injury: a rare presentation. Plast Aesthet Res 2015;2:85-7.

Source of Support: Nil, Conflict of Interest: None declared. Received: 29-08-2014; Accepted: 13-10-2014 